\title{
Prevenção da doença renal crônica à luz da perspectiva de pessoas hipertensas
}

\author{
Prevention of chronic kidney disease from the perspective of hypertensive people \\ Prevención de la enfermedad renal crónica desde la perspectiva de las personas \\ hipertensas
}

Ubiratan Ribeiro Martins Neto ${ }^{1 *}$, Vivian Rahmeier Fietz ${ }^{1}$, Sandra Regina Imada Akimura1.

\begin{abstract}
RESUMO
Objetivo: Identificar estratégias para prevenção da doença renal crônica (DRC) a partir das percepções de usuários hipertensos, cadastrados na atenção básica. Métodos: Trata-se de uma pesquisa descritiva e exploratória com abordagem qualitativa. A amostra dos participantes foi obtida pelo método de conveniência até a saturação das informações. A pesquisa foi desenvolvida em uma Unidade Básica de Saúde (UBS), localizada na cidade de DouradosMato Grosso do Sul. A coleta dos dados foi realizada por meio de uma entrevista semiestruturada com 26 usuários hipertensos que foram analisados pela técnica de análise de conteúdo (BARDIN L, 2016). Resultados: Foram encontradas duas categorias, sendo elas: entrevista e diálogo para realizar prevenção da DRC e atividades educativas enquanto método para construção de hábitos saudáveis e adequados no controle da HA e prevenção da DRC. Considerações Finais: As atividades para prevenção da DRC devem estar pautadas em práticas educativas dialógicas, com o intuito de promover a conscientização sobre a DRC e sua relação com a hipertensão arterial. Além disso, notou-se ser importante utilizar a estratégia de ensino em saúde sobre o tratamento da doença hipertensiva considerando o contexto vivenciado pelos hipertensos.
\end{abstract}

Palavras-chave: Doença renal crônica, Hipertensão arterial, Prevenção, Doenças crônicas.

\begin{abstract}
Objective: To identify strategies for the prevention of chronic kidney disease (CKD) from the perceptions of hypertensive users, registered in primary care. Methods: This is a descriptive and exploratory research with a qualitative approach. The sample of participants was obtained using the convenience method until information saturation. The research was carried out in a Basic Health Unit (UBS), located in the city of Dourados-Mato Grosso do Sul. The data collection was carried out through a semi-structured interview with 26 hypertensive users who were analyzed using the content analysis technique (BARDIN L, 2016). Results: Two categories were found, namely: Interview and dialogue to carry out CKD Prevention and Educational activities as a method for building healthy and adequate habits in controlling $A H$ and preventing CKD. Final Considerations: Activities for the prevention of CKD must be guided by dialogical educational practices, with the aim of promoting awareness about CKD and its relationship with arterial hypertension. In addition, it was noted that it is important to use the health teaching strategy on the treatment of hypertensive disease considering the context experienced by hypertensive patients.
\end{abstract}

Keywords: Renal insufficiency chronic, Hypertension, Disease prevention, Chronic disease.

\section{RESUMEN}

Objetivo: Identificar estrategias para la prevención de la enfermedad renal crónica (ERC) a partir de las percepciones de los usuarios hipertensos, registrados en atención primaria. Métodos: Se trata de una investigación descriptiva y exploratoria con enfoque cualitativo. La muestra de los participantes se obtuvo por el método de conveniencia hasta la saturación de la información. La investigación se llevó a cabo en una Unidad Básica de Salud (UBS), ubicada en la ciudad de Dourados-Mato Grosso do Sul. La recolección de datos se realizó mediante entrevista semiestructurada a 26 usuarios hipertensos que fueron analizados mediante la técnica de análisis de contenido (BARDIN L, 2016). Resultados: Se encontraron dos categorías, a saber: Entrevista y diálogo para la realización de actividades de Prevención de la ERC y Actividades Educativas como método para la construcción de hábitos saludables y adecuados en el control de la HA y la prevención de la ERC. Consideraciones finales: Las actividades para la prevención de la ERC deben estar guiadas por prácticas educativas dialógicas, con el objetivo de promover la conciencia sobre la ERC y su relación con la hipertensión arterial. Además, se señaló que es importante utilizar la estrategia de enseñanza de la salud en el tratamiento de la enfermedad hipertensiva considerando el contexto que viven los pacientes hipertensos.

Palabras clave: Fallo renal crónico, Hipertensión, Prevención de enfermedades, Enfermedad crónica.

${ }^{1}$ Universidade Estadual de Mato Grosso do Sul (UEMS), Dourados - MS. *E-mail: ubiratan41@gmail.com 


\section{INTRODUÇÃO}

O rim é responsável por diversas funções para a manutenção do organismo humano, como a excreção de resíduos metabólicos que não são utilizados pelo corpo, e se estes estiverem presentes no sangue em grandes quantidades podem acarretar sérios prejuízos (EATON DC e POOLER JP, 2015). Associa-se a diminuição da função renal com o aumento das taxas de morbimortalidade e diminuição da qualidade de vida (MALTA DC, et al., 2019).

$\mathrm{Na}$ doença renal crônica (DRC) ocorre o comprometimento da função renal de modo progressivo e sem capacidade de reversão, sendo acompanhado por uma lesão irreversível nas células do rim. Desta forma, acumula-se no sangue resíduos metabólitos como a creatinina, ácido úrico, metabólicos hormonais, ureia e urobilina, que é derivada do processo de degradação das células vermelhas, dentre outras (MEIRA SM, 2017).

A prevalência autorreferida da DRC de acordo com a análise dos dados da Pesquisa Nacional de Saúde (PNS) foi de 1,42\% nos adultos maiores de 18 anos, ou seja, em média dois milhões de brasileiros, sendo $1,48 \%$ no sexo feminino e 1,35 no sexo masculino. Em pessoas com mais de 65 anos a prevalência foi de 3,13\% (AGUIAR LK, et al., 2020).

A maior consequência da DRC está associada com sua evolução para a Doença Renal em Estágio Terminal (DRET) devido ao seu caráter progressivo. Neste estágio será preciso uma terapia renal substitutiva (TRS) para manter a vida, sendo representadas pela: hemodiálise, diálise peritoneal ou transplante renal (BRASIL, 2014).

O Sistema Único de Saúde (SUS) é responsável por manter $83 \%$ dos pacientes em TRS, sendo 91,4\% na modalidade de hemodiálise convencional, 0,9\% em hemodiálise diária e 7,7\% em diálise peritoneal. Os gastos com a DRET representaram 2,7 bilhões de reais no ano de 2016 (SBN, 2016).

A hipertensão arterial (HA) é a principal etiologia da DRC no Brasil, sendo que o número de pessoas, com mais de 18 anos e que apresentam diagnóstico dessa patologia renal, está em torno de 3 a 6 milhões (MARINHO AWGB, et al., 2017).

Em um estudo realizado com usuários hipertensos e diabéticos da atenção básica evidenciou-se que 17,3\% possuíam DRC e muitos precisariam ser acompanhados em unidades especializadas de nefrologia, entretanto, tais pessoas não tinham consciência acerca do diagnóstico e consequentemente não estavam em tratamento especializado (ALVES LF, et al., 2016).

A DRC, apesar de crônica, tem a possibilidade de ser evitada. No entanto, infelizmente os aspectos preventivos e de diagnóstico precoce ainda são realidades associadas com a não conscientização dos usuários da saúde, sobretudo entre os que possuem fatores de riscos para injúria renal. Soma-se a isso a dificuldade no acesso aos exames necessários (LUYCKX VA, et al., 2018).

Segundo Andrade TVF, et al. (2017), no caso da DRC é imprescindível desenvolver pesquisas e atividades voltadas para os grupos de risco como os hipertensos, no intuito de implementar estratégias preventivas e evitar a instalação e progressão da doença nesses indivíduos.

Dentro da atenção básica devem ser identificadas as principais vulnerabilidades e fatores de riscos modificáveis dos usuários hipertensos e assim planejarem e implementaram intervenções que possam evitar o desenvolvimento de comorbidades (DEUS FDF, et al., 2021).

Diante do exposto entendeu-se que a realidade da HA é marcada por pessoas suscetíveis a DRC e, de maneira geral, usuários da saúde podem não ter acesso apropriado às informações necessárias. Desta forma o objetivo do presente estudo foi identificar estratégias para prevenção da DRC à luz das percepções de usuários hipertensos da atenção básica.

\section{MÉTODOS}

Trata-se de uma pesquisa descritiva e exploratória com abordagem quantiqualitativa, sendo elencada a seguinte questão: Como prevenir a DRC a partir das percepções de pessoas hipertensas? A amostra dos participantes foi obtida pelo método da conveniência até a saturação das informações. 
A pesquisa foi desenvolvida na Unidade Básica de Saúde (UBS), localizada na cidade de Dourados-Mato Grosso do Sul. A coleta dos dados foi realizada com 26 usuários hipertensos e cadastrados no programa de hipertensão e diabetes (HIPERDIA). Foram selecionados usuários hipertensos para participarem desta pesquisa, pois, representam, no Brasil, o principal grupo de risco para evolução e desenvolvimento de DRC (BRASIL, 2014).

Os dados foram coletados nos meses de janeiro a março com 26 usuários, sendo 12 pessoas do gênero masculino e 14 do feminino a partir de entrevistas semiestruturada e audiogravadas, após os participantes assinarem o termo de consentimento livre e esclarecido (TCLE).

As entrevistas foram realizadas na UBS, em ambiente reservado dentro de um consultório de enfermagem, o que permitiu realizar uma abordagem individualizada e protegida e manteve a privacidade do participante. Para manutenção do anonimato da identidade de todos os participantes desta pesquisa foi realizada a identificação por meio da letra P, seguindo o exemplo: P1, P2, P3, P4, e assim sucessivamente. As falas dos entrevistados foram transcritas na íntegra e em seguida analisadas, conforme o referencial metodológico, de análise de conteúdo (BARDIN L, 2016).

A pesquisa esteve em conformidade com a resolução número 466 de 12 de dezembro de 2012, sendo encaminhada para a comissão de estágio, pesquisa, extensão e trabalho (CEPET) da secretaria municipal de saúde de Dourados. Após aprovada no CEPET, o projeto foi submetido na Plataforma Brasil, sendo aprovado pelo Comitê de Ética em Pesquisa com Seres Humanos (CESH) sob parecer 3.690.872 e precedeu em todas as etapas pela assinatura do TCLE.

\section{RESULTADOS E DISCUSSÃO}

Os 26 participantes hipertensos foram representados por $12(46,2 \%)$ pessoas do sexo masculino e 14 $(53,8 \%)$ do sexo feminino. A idade média geral dos participantes hipertensos foi de 65 anos e 6 meses, dos homens foi de 62 anos e seis meses e das mulheres 67 anos e cinco meses. A maioria dos participantes possuíam ensino fundamental incompleto $(53,8 \%)$, se autodeclararam brancos $(57,7 \%)$, religião católica $(61,5 \%)$. Os respectivos dados estão apresentados a seguir (Tabela 1).

Tabela 1 - Caracterização dos Hipertensos atendidos no Programa Hiperdia da UBS quanto aos critérios de idade, escolaridade, cor da pele e religião. Janeiro a Março de 2020, Dourados, MS.

\begin{tabular}{lcc}
\hline Variáveis & $\mathbf{N}$ & $\%$ \\
\hline Idade & 1 & $3,8 \%$ \\
\hline $30-40$ & 2 & $7,7 \%$ \\
$40-49$ & 5 & $19,2 \%$ \\
$50-59$ & 9 & $34,6 \%$ \\
$60-69$ & 8 & $30,8 \%$ \\
$70-79$ & 1 & $3,8 \%$ \\
$80-89$ & & \\
\hline Escolaridade & 1 & $3,8 \%$ \\
\hline Analfabeto & 14 & $53,8 \%$ \\
Ensino Fundamental Incompleto & 1 & $3,8 \%$ \\
Ensino Fundamental Completo & 7 & $26,9 \%$ \\
Ensino Médio Completo & 3 & $11,5 \%$ \\
Ensino Superior Completo & & \\
\hline Cor da Pele & 9 & $34,6 \%$ \\
\hline Pardo & 1 & $3,8 \%$ \\
Amarelo & 1 & $3,8 \%$ \\
Negro & 15 & $57,7 \%$ \\
Branco & & \\
\hline Religião & 16 & $61,5 \%$ \\
\hline Católico & 6 & $3,1 \%$ \\
Evangélico & 1 & $7,7 \%$ \\
Espírita & 2 & $3,8 \%$ \\
Agnóstico & 1 & \\
Testemunha e Jeová & & \\
\hline & & $20 \%$ \\
\hline
\end{tabular}

Fonte: Martins Neto UR, et al., 2021. 
Notou-se um predomínio de participantes com renda per capita entre um salário-mínimo e um saláriomínimo e meio (42,4\%), residiam em moradia própria (84,6\%) e são aposentados $(46,2 \%)$. Os dados estão apresentados a seguir (Tabela 2).

Tabela 2 - Caracterização dos Hipertensos atendidos no Programa Hiperdia da UBS quanto aos critérios de renda per capita, moradia e atividade ocupacional. Janeiro a Março de 2020, Dourados, MS.

\begin{tabular}{lcc}
\hline Variáveis & $\mathbf{N}$ & $\%$ \\
\hline Renda per capita & 3 & $11,5 \%$ \\
\hline Até $\mathrm{R} \$ 499,00$ & 7 & $26,9 \%$ \\
$>\mathrm{R} \$ 499,00$ e até $\mathrm{R} \$ 998,00$ & 11 & $42,3 \%$ \\
$>\mathrm{R} \$ 998,00$ e até $\mathrm{R} \$ 1497,00$ & 1 & $3,8 \%$ \\
$>\mathrm{R} \$ 1497,00$ e até $\mathrm{R} \$ 1996,00$ & 3 & $11,5 \%$ \\
$>\mathrm{R} \$ 1996,00$ e até $\mathrm{R} \$ 2994,00$ & 1 & $3,8 \%$ \\
$>\mathrm{R} \$ 2994,00$ e até $\mathrm{R} \$ 3992,00$ & & \\
\hline Moradia & 22 & $84,6 \%$ \\
\hline Própria & 4 & $15,4 \%$ \\
Alugada & & \\
\hline Atividade Ocupacional & 12 & $46,2 \%$ \\
\hline Aposentado & 4 & $15,4 \%$ \\
Do Lar & 2 & $7,6 \%$ \\
Sapateiro & 1 & $3,8 \%$ \\
Mecânico & 1 & $3,8 \%$ \\
Advogado & 1 & $3,8 \%$ \\
Motorista de Ônibus & 1 & $3,8 \%$ \\
Auxílio-Doença & 1 & $3,8 \%$ \\
Taxista & 1 & $3,8 \%$ \\
Auxiliar de pedreiro & 1 & $3,8 \%$ \\
Auxiliar de limpeza & 1 & $3,8 \%$ \\
Pensionista & 1 &
\end{tabular}

Fonte: Martins Neto UR, et al., 2021.

A análise das entrevistas subsidiou para o levantamento de duas categorias, sendo elas: entrevista e diálogo para realizar prevenção da DRC e atividades educativas enquanto método para construção de hábitos saudáveis e adequados no controle da HA e prevenção da DRC. As duas categorias foram subdividida em outras duas subcategorias, e estão apresentadas a seguir (Quadro 1).

Quadro 1 - Categorias e subcategorias encontradas por meio da análise das entrevistas. Janeiro a Março de 2020, Dourados, MS.

\begin{tabular}{|l|l|}
\hline \multicolumn{1}{|c|}{ Categoria } & \multicolumn{1}{c|}{ Subcategorias } \\
\hline \multirow{2}{*}{$\begin{array}{l}\text { Entrevista e diálogo para realizar prevenção da } \\
\text { DRC }\end{array}$} & $\begin{array}{l}\text { Realizar questionamentos e permitir elucidações } \\
\text { sobre a DRC e suas consequências. }\end{array}$ \\
\cline { 2 - 2 } Fazer correlações entre a HA e a DRC \\
\hline \multirow{2}{*}{$\begin{array}{l}\text { Atividades educativas enquanto método para } \\
\text { construção de hábitos saudáveis e adequados } \\
\text { no controle da HA e prevenção da DRC }\end{array}$} & $\begin{array}{l}\text { Informar acerca do tratamento medicamentoso } \\
\text { de maneira racional. }\end{array}$ \\
\cline { 2 - 2 } & $\begin{array}{l}\text { Criar ferramentas para ensinar sobre a } \\
\text { necessidade e importância do tratamento não- } \\
\text { medicamentoso e a importância dos hábitos de } \\
\text { vida adequados (alimentação, atividade física e } \\
\text { manejo do estresse). }\end{array}$ \\
\hline
\end{tabular}

Fonte: Martins Neto UR, et al., 2021.

\section{Entrevista e diálogo para realizar Prevenção da DRC}

Na realização da entrevista em formato de diálogo, os hipertensos começaram a realizar questionamentos e, assim, percebeu-se que os participantes apresentaram um conhecimento incipiente em relação a DRC e suas consequências para o organismo. Além disso, não faziam associação da doença hipertensiva com patologia renal. 
Nesse sentido a análise evidenciou, duas subcategorias que convergiram com o tema, sendo eles: realizar questionamentos e permitir elucidações sobre a DRC e suas consequências e fazer correlações entre a HA e a DRC. As subcategorias serão apresentadas a seguir.

\section{Realizar questionamentos e permitir elucidações sobre a DRC e suas consequências}

A análise das entrevistas evidenciou que a DRC ainda é pouco compreendida por parte dos entrevistados deste estudo, sendo este bem superficial. Na sequência serão exibidas falas que deram origem a formação desta subcategoria ao perguntarem aos participantes suas respectivas compreensões acerca da DRC:

"Doença Renal? O que eu conheço? É pressão e enfermidade por dentro né? Dor, sente muita dor por dentro. Dor nas costa e coluna. Tudo que ataca por dentro é renal. Dores que eu tenho no pé da virilha. Tudo essas dores que ataca por dentro. Por fora eu não tenho nada" (P1).

"Eu não conheço nada dela. Eu sei que ela tranca o rim. Só isso. Eu não sei sobre ela" (P4).

"Olha essa doença renal eu não conheço bem ela, sabe [...]" (P5).

"Bom, isso ai eu não to conhecendo nada né, porque a gente não tem essa doença" (P10).

"Eu não conheço nada. Nada. Do rim nada. (P11).

"O que eu entendo Rapaz, eu sou leigo nesse negócio, porque eu não tenho estudo né. Eu só sei que é sofrido demais" (P23).

Pode-se observar que houve um predomínio da expressão "não conheço" ou até pedindo uma explicação e, assim, percebeu-se claramente o conhecimento incipiente acerca da DRC por parte dos entrevistados hipertensos.

Deste modo é indispensável elaborar estratégias de educação em saúde para prevenção da DRC (SANTOS JRFM, et al., 2017). Sendo importante que essas práticas educativas sejam implementadas junto aos usuários dos grupos de riscos para desenvolverem a patologia renal na atenção básica (DALLACOSTA FM, et al., 2017). A HA é o maior fator de risco no Brasil para ocasionar a DRC (SOARES FC, et al., 2017). $\mathrm{Na}$ assistência as pessoas com doenças crônicas, é essencial uma abordagem que valorize a realidade dos indivíduos e que propicie o seu protagonismo para obter a prevenção de agravos (BRASIL, 2014).

Assim, como foi notado em relação ao conhecimento sobre a DRC por parte das pessoas hipertensas desse estudo, percebeu-se também que as consequências da patologia renal não são bem elucidadas por parte dos participantes.

A seguir serão apresentados as falam que foram agrupadas e formaram esta parte da subcategoria. A maioria das respostas abaixo foram obtidas quando se perguntou aos participantes como eles compreendiam as consequências da DRC.

“[...] Ela pode causar alteração e pressão de sangue e urina, mas eu não sei bem o que causa assim, eu não sei o que é não [...]" (P4).

"[...] Ah, eu sei que é uma doença grave que da muitos problemas, inclusive acho que do coração essas coisas, mas não sei mais te falar alguma coisa mais [...]" (P8).

"[...] Ah, eu nem sei qual é a consequência, assim porque na minha família não teve ninguém ainda que teve assim [...]" (P19).

“[...] Ah, você me pegou no pulo agora (risadas). Da problema de câncer? Os rins? Para os rins se não toma água? É? Que mais? Não pode tomar muita bebida de álcool porque prejudica e da inchaço é? Então só isso que eu entendo [...]" (P21).

"[...] Não sei, mas geralmente dizem que a pele fica, o olho fica amarelado, fica sintomas. Mas eu assim, o que acontece assim eu não tenho ideia do que é, sei que fica o olho assim, porque já vi entrevista falando de alguns sintomas assim. Mas exatamente o que é, sintomas e o que pode causar, eu não sei [...]" (P25). 
A expressão "não sei" foi um destaque nas entrevistas apresentadas. Também pode-se notar que o pedido por explicações sobre o tema veio incorporado na fala dos participantes, sendo assim entende-se tanto a DRC (primeira subcategoria), como suas consequências são necessidades a serem trabalhadas.

A DRC representa um grave problema de saúde e tem aumentado em todo mundo, sendo que no Brasil existe uma grande dificuldade em realizar o tratamento na atenção básica (MELO APM, et al., 2013). Dessa maneira deve-se investir na educação em saúde para prevenir a instalação e progressão da patologia renal crônica (SANTOS JRFM, et al., 2017). Além dos aspectos preventivos da injúria renal, a educação em saúde, tende a subtrair os elevados gastos no SUS e aumentar a qualidade de vida das pessoas que realizam tratamento da HA (MENEZES ACMP e GOBBI D, 2010).

\section{Fazer correlações entre a HA e a DRC}

Conforme a análise evidenciou, os participantes não conhecem sobre a DRC e nem suas consequências no organismo humano e, sobretudo, percebeu-se que os mesmos também não conseguem, de maneira clara, estabelecer relação da Hipertensão com a DRC.

Em seguida serão exibidas falas dos hipertensos quando o pesquisador perguntou sobre o conhecimento da existência de uma correlação entre a HA e a DRC.

"Não tenho a mínima ideia. Eu não sei como é que leva, eu sei que ela leva né, mas eu não sei porque que ela leva" (P4).

"Pressão alta. Então, por causa da pressão, é por causa do, como que fala? Por causa renal. Por causa do rim que sobe a pressão?” (P6).

"Qual relação? Não sei te falar" (P8).

"Então, eu não tenho entendimento assim, sobre a pressão que afeta o rim. Então, eu não tenho esse conhecimento até hoje né" (P16).

"Não sei viu. É relacionado os dois? É? (risadas)" (P20).

Em um estudo desenvolvido entre 448 usuários do HIPERDIA em municípios do Meio Oeste de Santa Catarina, sendo 376 hipertensos, os autores identificaram que 110 pessoas estavam com a taxa de filtração glomerular $>60 \mathrm{ml} / \mathrm{min}$, ou seja, com grande possibilidade de possuírem a DRC. Sendo assim, torna-se importante desenvolver medidas de educação em saúde a população de risco, como os hipertensos, no sentido de prevenir o desenvolvimento ou, ainda, a evolução da DRC (DALLACOSTA FM, et al., 2018).

Reconhecer as áreas de maior vulnerabilidade, auxilia na atuação das ESF e na elaboração de intervenções eficientes capazes de reduzir os riscos e cooperar com o reestabelecimento salutar da comunidade

As medidas preventivas sobre a DRC com os hipertensos devem pautar-se em uma abordagem que permita o protagonismo das pessoas com HA (DUARTE GC, et al., 2015). O uso de métodos de ensino dialógicos, alinhado ao contexto vivenciado pelo indivíduo, contribui com as mudanças no estilo de vida, visto que ocorre uma troca de conhecimentos crítico-reflexiva entre os usuários e profissionais de saúde (ALMEIDA ER, et al., 2014). O modelo de educação tradicional é denominado prática bancária e o intuito da educação é libertar-se dessa prática, ou seja, sem depositar conteúdos desarticulados com o contexto real (FREIRE P, 2015).

\section{Atividades educativas enquanto método para construção de hábitos saudáveis e adequados no controle da HA e prevenção da DRC}

A presente categoria foi construída considerando que diversos fatores contribuem para a HA estar associada enquanto etiologia e desenvolvimento da DRC. Assim, percebeu-se a necessidade de informar e criar ferramentas para que as informações possam chegar aos hipertensos e minimizar os efeitos e o avanço da doença. 
Embora os entrevistados relatarem sobre a importância do uso racional dos medicamentos e a necessidade em estabelecer hábitos de vida saudáveis, os mesmos não conseguiram associar o tratamento medicamentoso e não-medicamentoso da doença hipertensiva com a prevenção da DRC e, ainda realizar o controle dos níveis pressóricos.

\section{Informar sobre o tratamento medicamentoso de maneira racional}

Durante as entrevistas ao perguntar aos participantes sobre suas respectivas dificuldades e dúvidas frente ao tratamento da HA, notou-se que todos relacionavam tratamento ao uso da medicação anti-hipertensiva.

O uso correto da medicação é extremamente importante para o controle da HA e prevenção de complicações, entretanto o tratamento também inclui medidas que envolvem os hábitos de vida como o IMC apropriado, atividade física, alimentação saudável, controle da circunferência da cintura, evitar o tabagismo e o consumo excessivo de álcool (BRASIL, 2014; SBC, 2016).

A seguir serão exibidos trechos das entrevistas em que os participantes associam o tratamento apenas a medicação:

"Não. Depois que eu comecei tratar tomar essa losartana e esses remédios da pressão não deu problema mais em mim. Eu não tenho dúvida né, tomando o remédio eu acho que controla, ta em ordem né" (P4).

"Não não. não tenho não. Porque eu tomo o remédio certo né? Eu não tenho dúvida não" (P5).

“[...] Tratando, tratando com remédio e não descuidar. Fazer sempre o tratamento" (P9).

"Até agora não. Eu acho que tem que ser tratada né. O único jeito é você toma o medicamento e controla a pressão que não é brincadeira. Se não cuida é isso" (P16).

"Não, dificuldade não teve, porque ai eu vim no médico aqui né, passava la o losartana, media a pressão e fazia o acompanhamento. E ai você precisa fazer um acompanhamento né. E por enquanto ela está sempre doze por oito, treze por oito, treze por novo. Vive nessa faixa" (P26).

Pode-se observar que a maioria dos entrevistados consideram fácil o tratamento da HA. A conjuntura observada reflete o olhar dos participantes em relação ao HIPERDIA, sendo representado por uma renovação de receita, uma vez que não foi possível observar os participantes relatando suas necessidades em relação ao tratamento não-medicamentoso frente aos determinantes e condicionantes da saúde.

Outro fator importante, relacionado com o agravamento da HA, pode ser o grau de escolaridade baixo, que está relacionado com a baixa adesão ao tratamento medicamentoso e não medicamentoso e no mais essa condição associa-se as baixas condições financeiras para efetuar o tratamento. Desta forma, investir na escolaridade e educação da população contribui com a saúde dos cidadãos (MILL JG, 2019).

Criar ferramentas para ensinar sobre a necessidade e importância do tratamento não- medicamentoso e a importância dos hábitos de vida adequados (alimentação, atividade física e manejo do estresse)

Como foi mencionado, na subcategoria anterior, os participantes associam o tratamento exclusivamente aos medicamentos e não apontaram, diretamente, suas necessidades frente a implementação e manutenção dos hábitos de vida saudáveis para tratar a HA e consequentemente prevenir as complicações.

Os participantes relataram sobre o que seria importante abordar em um processo educativo para pessoas com HA. Evidenciou-se o anseio em conhecer sobre alimentação saudável e a prática de exercício físico.A seguir serão apresentados os trechos da entrevista que convergiram para a construção da presente subcategoria:

"Fazer dieta, fazer uma dieta, não comer sal, comida salgada, água, água também de qualidade e filtrada [...] Fui medi e foi preciso vim, mas foi num velório isso dai que ela subiu assim 17 ai tive que vim embora. Passei no hospital que o posto tava fechado que era um domingo. Mediram e colocaram um comprimido em baixo da língua e eu vim embora. Acho que é emoção por exemplo" (P3). 
"Tem que falar sobre alimentação. Principalmente alimentação né. O sal, evitar o sal e gordura né. A alimentação como melhorar uma alimentação mais saudável, mais verdura" (P12).

"Se eu tiver assim, um negócio chega e da uma notícia assim que me preocupa, mas na hora minha pressão sobe. Esses dias eu fui fazer o cateterismo ali no Evangélico, fui 3 vezes e quando eu deitava na mesa minha pressão no mesmo momento ia para 17. Duas vezes, três vezes eu voltei do hospital, fui para casa, ai depois voltava de novo e na terceira vez deu certo" (P13).

"É muito bom, porque tem muito que não sabe. É bom falar do alimento primeiro principalmente, porque eu tenho minha amiga, aquela lá eu acho que tinha que trazer um desenho e falar (risadas), porque olha eu vejo ela, ela várias vezes caiu com a pressão alta, mas o jeito que ela come, só por Deus mesmo para ter misericórdia” (P14).

As falas dos participantes apontaram que os mesmos têm anseios em aprender sobre como se alimentarem de modo saudável e também praticar atividade física. Além disso, os pacientes mencionaram que deveriam realizar controle mais sistemático em relação aos níveis pressóricos, visto que a hipertensão descontrolada pode provocar a DRC e até mesmo outras doenças.

Além disso, os participantes relataram aleatoriamente como o estresse psicológico influencia negativamente nos níveis pressóricos. Um estudo mostrou que os indivíduos com a patologia hipertensiva são 9,7 vezes mais estressados que os indivíduos que não têm esse diagnóstico (MOXOTÓ GFA, et al., 2015).

Nas pessoas com estresse de origem psicológica aguda e que possuem elevação da pressão arterial é preciso otimizar a medicação anti-hipertensiva e também conscientizar sobre a adesão ao tratamento (SBC, 2016).

\section{CONSIDERAÇÕES FINAIS}

O presente estudo desenvolvido com usuários hipertensos acompanhados no HIPERDIA desvelou estratégias para prevenção da DRC com pessoas hipertensas. Sendo identificado sobre a importância das práticas educativas dialógicas entre profissionais de saúde e usuários hipertensos para promover uma conscientização sobre a DRC e sua relação com a HA. Além disso, verificou-se a importância do ensino em saúde, sobretudo para esse público de hipertensos do HIPERDIA. Percebeu-se que as principais questões a serem desenvolvidas seriam sobre o tratamento medicamentoso e não medicamentoso da doença hipertensiva considerando o contexto vivenciado. Além disso, notou-se a necessidade de realizar atividades que incentivem ao uso correto das medicações anti-hipertensivas e a adesão aos hábitos de vida saudáveis.

\section{AGRADECIMENTOS}

Ao Programa Institucional de Bolsas aos Alunos de Pós-Graduação (PIBAP) da Universidade Estadual de Mato Grosso do Sul (PIBAP/UEMS) que concedeu bolsa de estudo e estágio docência para um dos autores desta pesquisa sob Edital № 02/2019- PPGES/UEMS, de 09 de abril de 2019.

\section{REFERÊNCIAS}

1. AGUIAR LK, et al. Fatores associados à doença renal crônica: inquérito epidemiológico da Pesquisa Nacional de Saúde. Rev. Bras. Epidemiol,2020; 23(5): E200044.

2. ALMEIDA ER, et al. A prática da educação em saúde na percepção dos usuários hipertensos e diabéticos. Saúde Debate, 2014; 38(101): 328-337.

3. ALVES LF, et al. Prevalência da doença renal crônica em um município do sudeste do Brasil. Jornal Brasileiro de Nefrologia, 2017; 39(2): 126-134.

4. ANDRADE TVF, et al. Avaliação da função renal em pacientes portadores de doenças crônicas não transmissíveis. Revista de Biologia Farmácia e Manejo Agrícola, 2017; 13(3): 34-38. 
5. BARDIN L. Análise de conteúdo. 1. ed. São Paulo: Edições 70, 2016; 279p.

6. BRASIL. Diretrizes clínicas para o cuidado ao paciente com doença renal crônica - DRC no sistema único de saúde. Brasília. 2014. Disponível em: https://portalarquivos2.saude.gov.br/images/pdf/2014/marco/24/diretriz-cl--nica-drcversao-final.pdf. Acesso em: 15 abr. 2020.

7. BRASIL. Estratégias para o cuidado da pessoa com doença crônica. Brasília. 2014. Disponível em: https://bvsms.saude.gov.br/bvs/publicacoes/estrategias_cuidado_pessoa_doenca_cronica_cab35.pdf. Acesso em: 18 abr. 2020.

8. DALLACOSTA FM, et al. Detecção precoce de doença renal crônica em população de risco. Cogitare Enfermagem, 2017; 22(2): e48714.

9. DALLACOSTA FM, et al. Doença renal: detecção em grupos de Hiperdia. Rev. Saúde.Com, 2018; 14(2): $1197-1201$.

10. DEUS FDF, et al. Estratificação do risco cardiovascular em pacientes hipertensos de um município do interior de Minas Gerais. Revista Eletrônica Acervo Saúde, 2021; 14(5): e6981.

11. DUARTE GC, et al. Práticas de promoção à saúde e prevenção de agravos no grupo hiperdia. Revista Espaço Ciência e Saúde. Cruz Alta, 2015; 3(1): 59-69.

12. EATON DC, POOLER JP. Vander's renal physiology. 8. ed. New York: MC Graw Hill, 2015; 224p.

13. FREIRE P. Pedagogia da autonomia: saberes necessários à prática educativa. 51. ed. Rio de Janeiro: Paz e Terra, 2015; 143p.

14. LUYCKX VA, et al. The global burden of kidney disease and the sustainable development goals. Bull World Health Organ, 2018; 96(6): 414-422.

15. MALTA DC, et al. Avaliação da função renal na população adulta brasileira, segundo critérios laboratoriais da Pesquisa Nacional de Saúde. Rev. Bras. Epidemiol, 2019; 22(2): E190010.

16. MARINHO AWGB, et al. Prevalência de doença renal crônica em adultos no Brasil: revisão sistemática de literatura. Caderno de Saúde Coletiva, 2017; 25(3): 379-388.

17. MEIRA SM. Doença Renal Crônica. Ciência e Saúde, 2017; 10(3): 120.

18. MELO APM, et al. Diagnóstico precoce da doença renal crônica pela Estratégia de Saúde da Família. Revista Interdisciplinar, 2013; 6(1): 124-128.

19. MENEZES ACMP, GOBBI D. Educação em saúde e Programa de Saúde da Família: atuação da enfermagem na prevenção de complicações em pacientes hipertensos. O Mundo da Saúde, 2010; 34(1): 97-102.

20. MILL JG. Determinantes sociais na hipertensão arterial. Arq. Bras. Cardiol, 2019; 113(4): 696-698, 2019.

21. MOXOTÓ GFA, et. al. Raiva, stress emocional e hipertensão: um estudo comparativo. Psic.: Teor. e Pesq, 2015, 31(2): 221-227.

22. SANTOS JRFM, et al. Estratégias da atenção básica na doença renal crônica: a importância do diagnóstico precoce. Revista de Saúde.Com, 2017; 13(2): 863-870.

23. SOARES FC, et al. Prevalência de hipertensão arterial e diabetes mellitus em portadores de doença renal crônica em tratamento conservador do serviço ubaense de nefrologia. Revista Científica Fagoc - Saúde, 2017; 2(2): 21-26.

24. SOCIEDADE BRASILEIRA DE CARDIOLOGIA (SBC). VII diretriz brasileira de hipertensão arterial. Arquivos

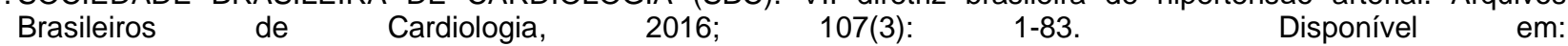
http://publicacoes.cardiol.br/2014/diretrizes/2016/05_HIPERTENSAO_ARTERIAL.pdf

25. SOCIEDADE BRASILEIRA DE NEFROLOGIA. Diálise no Brasil: cenário atual e desafios. 2017; 47p. Disponível em: https://arquivos.sbn.org.br/uploads/HDU-DRA-CARMEM-TZANNO.pdf. 